\title{
Letter to Editor: Challenges of Implementing Disaster Preparedness Plan in Iran Hospitals
}

ditation: Masoumi GhR, Dehghani A. Challenges of Implementing Disaster Preparedness Plan in Iran Hospitals. Health in Emergencies and Disasters Quarterly. 2016; 2(1):3-4.

\section{Introduction}

The global investigation in recent years indicates that the number of disasters has increased annually with more people affected and higher financial losses, too [1]. Disasters by threatening people's lives affect different aspects of society, including health, economy, policies, and international relations [2]. More than $90 \%$ of deaths caused by disasters occur in developing countries and unsustainable development in these countries is partly due to the occurrence of different accidents and disasters. In the past 90 years, more than 180000 people lost their lives due to natural disasters with a lot of physical, psychological, and social consequences [3].

Structural, non-structural, and functional components of hospitals can be affected by disasters. Besides injuries to staff and patients, as well as damages to hospital properties and equipment, the performance of hospital is lost for admission of injured people and therefore the mortality rate caused by accidents will increase [4].

\section{Hospital Preparedness Plan}

Hospitals play important roles in both predictable and unpredictable emergencies. In addition, disaster preparedness plan in hospitals requires careful programing [5]. Planning for preparation in disasters demands valid and systematic data of the disaster outcomes and awareness of policymakers of all stages of a disaster [6]. Hospitals as the first line of health care services in disasters must be prepared to deal with them. A precise and effective disaster preparedness for staff of hospitals should be prepared and implemented before the disaster and run continually [7].

Hospital preparedness in disasters should be comprehensive and not to create problems in hospital performance during accidents and disasters. Accordingly, hospitals should be ready with regard to different aspects of their operations before the events occur [8]. Hospital preparedness plan should be compiled and updated based on the type of the event, its probability and topology, how to serve the hospital, human resources, financial resources, equipment and patient admissions [9]. Preparation should be considered in all respects as physical structure, administrative structure, equipment, manpower, and so on [10].

\section{Challenges}

Based on Ministry of Health and Medical Education report (Table 1), many Iranian hospitals are very old and replacement with new ones is essential, especially, focusing on non-structural parts is crucial.

According to Ardalan et al. study (2014) on 224 hospitals, disasters preparedness in Iranian hospitals was not acceptable and most of them need improvement. Based on this study, the average (SD) score of all safety components was 32.4(12.7) out of 100. A total of 122(54.5\%) hospitals were classified as having low safety and $102(45.5 \%)$ hospitals as having average safety. No hospital was placed in the high safety category.

The most challenging part is implementing hospital disaster risk reduction (HDRR) program and hospital disaster plan (HDP) or some other relevant programs in hospitals. One of the most important weak points is that HDP or HDRR is not a priority in hospitals, especially compared to economic or administrative problems. Therefore, most hospital managers are not interested to run this program.

Moreover, there are internal barriers such as the high costs of plan implementation, absence of common language, and lack of empowerment, knowledge, or motivation in hospital managers and staff, in short no desire for crisis management.

\section{Solution}

Ardalan et al. recommended that a disaster preparedness plan in hospitals should have these components: 1) establishing a national crisis committee in hospitals, 2) explanation of hospital safety indexes in hospitals under construction and development, 3) planning structural and non-structural safety and preparedness programs in 
hospitals and over their performances, 4) define indicators of readiness and safety of hospitals in the National Hospital Accreditation [11].

According to literature review, we should implement HDRR and HDP and organize requirements with preparation of training programs, creation of interests in personnel, designing protocols and job description, encouraging staff and managers and so on.

\section{Conclusion}

Ministry of Health and Medical Education has started to consider these recommendations by inserting disaster and risk reduction part in hospital accreditation checklists. However, it is not enough. Actually, we have national guidebook for HDP and we are going to have HDRR in revision of HDP.

Sufficient budget is necessary to establish communication and technical infrastructures along with emergency medical equipment in hospitals for processing appropriate services at times of disasters.

Also, holding up safety training courses for medical personnel, which are related to their daily work with disaster approach, could be more effective than traditional education. Using protocols and hospital emergency operation plan certainly increases hospital performance in disasters. However, incoordination between hospital wards, logistics, and supportive systems is one of the key points, that makes HDP implementation is too difficult in hospitals. Interventional studies along with qualitative ones is essential for choosing the best plan for improving hospital disaster risk reduction.

\section{Gholamreza Masoumi $^{1 *}$, Arezoo Dehghani}

1. Department of Emergency Medicine, Emergency Medicine Research Center, Iran University of Medical Sciences, Tehran, Iran.

2. Department of Medical Education, School of Medical Education Sciences, Shahid Beheshti University of Medical Sciences, Tehran, Iran.

* Corresponding Author:

Gholamreza Masoumi, PhD

Address: Department of Emergency Medicine, Emergency Medicine Research Center, Iran University of Medical Sciences, Tehran, Iran. E-mail: greza.masoumi@gmail.com

\section{References}

[1] Coppola DP. Introduction to international disaster management.Oxford: Springer; 2011.

[2] Nasiri-pour A, Raeissi P, Mahbobi M. [Border hospital readiness in handling border related crisis in Kermanshah Province, Iran, 2007 (Persian)]. Journal of Health Administration. 2007; 10(28):41-48

[3] Khankeh HR. [Hospital preparedness for emergency events; Country plan (Persian)]. University of Social Welfare and Rehabilitation Sciences; 2012

[4] Ardalan A, Sabz Ghabaei A, Najafi A, Zonoubi V. [Assessment of disaster risk in the hospital (Persian)]. Tehran: Movaffagh Publication; 2010.

[5] Ojaghi Sh, Nourizadeh S, Mahboubi M, Khazaei AAR, Najafi GhA. [Disaster crisis handling preparedness level of hospitals in Kermanshah (Persian)]. Journal of Kermanshah University Of Medical Sciences (Behbood). 2009; 13(3):267:274.

[6] Guha-Sapir D, Below R. The quality and accuracy of disaster data: A comparative analyses of three global data sets. Washinghton, D.C.: ProVention Consortium, Disaster Management Facility; 2002.

[7] Smith SM, Gorski J, Vennelakanti HC. Disaster preparedness and response: a challenge for hospitals in earthquakeprone countries. International Journal of Emergency Management. 2010; 7(3-4):209. doi: 10.1504/ijem.2010.037006

[8] Malekshahi F, Mardani M. Abilities and limitations of crisis management in Shohadaye Ashayer and Social Security hospitals of Khorramabad in 2007. Iranian Journal of Critical Care Nursing. 2009; 1(1):29-34

[9] Zaboli R, Toufighi Sh, Seyyedin SH, Malmoon Z, Hosseini Shokuh SM. Organizational vulnerability and management of clinical departments against crisis. Iranian Journal of Critical Care Nursing. 2009; 2(3):99-103.

[10] Arab M, Zeraati H, Akbari Haghighi F, Ravangard R. [A study on the executive managers' knowledge and performance, and their hospitals preparedness against earthquake events and their relationships at public hospitals (affiliated by Tehran University of Medical Sciences (TUMS) 2005-2006) (Persian)]. Journal of Health Administration. 2009; 11(34): 7-14.

[11] Ardalan A, Kandi Keleh M, Saberinia A, Khorasani-Zavareh D, Khankeh H, Miadfar J, et al. 2015 estimation of hospitals safety from disasters in I.R.Iran: The Results from the Assessment of 421 Hospitals. Plos One. 2016; 11(9):0161542. doi: 10.1371/journal.pone.0161542 